\title{
An Axisymmetric Squeezing Fluid Flow between the Two Infinite Parallel Plates in a Porous Medium Channel
}

\author{
S. Islam, ${ }^{1}$ Hamid Khan, ${ }^{2}$ Inayat Ali Shah, ${ }^{2}$ and Gul Zaman ${ }^{3}$ \\ ${ }^{1}$ COMSATS Institute of Information Technology, Chakshazad, Islamabad 44000, Pakistan \\ ${ }^{2}$ Islamia College Peshawar (Chartered University), Khyber Pakhtunkhwa, Peshawar 25120, Pakistan \\ ${ }^{3}$ Malakand University, Khyber Pukhtonnkhawa, Chakdara 1800, Pakistan \\ Correspondence should be addressed to S. Islam, saeed@comsats.edu.pk
}

Received 5 October 2010; Revised 14 December 2010; Accepted 22 February 2011

Academic Editor: Jaromir Horacek

Copyright (C) 2011 S. Islam et al. This is an open access article distributed under the Creative Commons Attribution License, which permits unrestricted use, distribution, and reproduction in any medium, provided the original work is properly cited.

The flow between two large parallel plates approaching each other symmetrically in a porous medium is studied. The Navier-Stokes equations have been transformed into an ordinary nonlinear differential equation using a transformation $\psi(r, z)=r^{2} F(z)$. Solution to the problem is obtained by using differential transform method (DTM) by varying different Newtonian fluid parameters and permeability of the porous medium. Result for the stream function is presented. Validity of the solutions is confirmed by evaluating the residual in each case, and the proposed scheme gives excellent and reliable results. The influence of different parameters on the flow has been discussed and presented through graphs.

\section{Introduction}

The study through a porous medium is an interesting and hot issue in these days, especially, with the introduction of the modified Darcy's Law [1], in contrast to the classical Darcy's Law [2] and the Brinkman model [3]. The flow through a porous media has wide spread applications in engineering and science, such as ground water hydrology, petroleum engineering, reservoir engineering, chemical engineering, chemical reactors to agriculture irrigation and drainage and the recovery of crude oil from the pores of the reservoir rocks [4-9].

Squeezing flows are common in moulding, food industry, and chemical engineering, and they have, therefore, been studied for a long time as researchers and scientists have sought to optimize processing operations to produce improved components. These flows are 
also interesting from rheological perspective [10]. The fundamental analysis of the squeezing flow between two large parallel plates was carried out by Stefan [11] in which he developed an ad hoc asymptotic solution for a thin film of Newtonian fluid. Naduvinamani et al. in $[12,13]$ studied static and dynamic behavior of squeeze-film lubrication of narrow porous journal bearings with coupled stress fluid and a squeeze film lubrication of a short porous journal bearing with couple stress fluids, respectively.

The determination of squeeze flow characteristics has attracted the attention of several investigators due to its importance in the practical problems of improving the performance of hydraulic machine elements, food industry, chemical engineering, polymer processing, compression, and injection molding [14-20].

Differential transform method (DTM) is one of the numerical methods for differential equations. The basic idea of differential transformation was initially introduced by Zhou [21] in 1986. Its main application therein is to solve both linear and nonlinear initial value problems in electrical circuit analysis. This method constructs the solution in the form of a polynomial. It is different from the traditional higher-order Taylor series method. The DTM is an alternative procedure for getting Taylor series solution of the differential equations. This method reduces the size of computational domain and is easily applicable to a variety of problems. The method was applied to differential algebraic equations (DAEs) of index1 by Ayaz [22]. Liu and Song [23] analyzed higher index differential algebraic equations using this technique where they showed that the method is effective in case of index-2 DAEs but not suitable for DAEs of index-3. Application of the two-dimensional differential transform method was studied by Ayaz [24] for partial differential equations. Comparison of this method with ADM was done by Hassan [25] to solve PDEs. The same author has used this method to solve higher-order initial value problems where he studied second- and thirdorder initial value problems to show the efficiency of the method. Islam et al. [26] used this method for the solution of special twelfth-order boundary value problems.

The study reported in this paper considers the axisymmetric squeezing flow of a viscous fluid between two large parallel plates in a porous medium separated by a small distance $2 \mathrm{H}$ and the plates approaching each other with a low constant velocity $\mathrm{V}$, and the flow can be assumed to be quasisteady. We use DTM to find the approximate solutions of the modeled problem.

\section{Basic Equations and Problem Formulation}

Employing the modified Darcy's Law, the two-dimensional flow of an incompressible axisymmetric homogenous fluid in a porous medium in the absence of body force is governed by the following equations $[27,28]$ :

$$
\begin{gathered}
\nabla \cdot u=0 \\
\rho \frac{d u}{d t}=\nabla \cdot T+r,
\end{gathered}
$$

where $u=\left[u_{r}(r, z), 0, u_{z}(r, z)\right]$ is the velocity vector, $d / d t$ denotes the material time derivative, $\rho$ is the constant density, $T$ is the Cauchy stress tensor where $T=-p I+\mu A_{1}$ in which 
$A_{1}=\nabla u+(\nabla u)^{T}$ is the Rivlin-Ericksen tensor, $\rho$ is the pressure, $\mu$ is the viscosity, and $r$ is the Darcy's resistance given by the relation,

$$
r=-\frac{\bar{\mu} u_{d}}{k}
$$

here, $k$ is the permeability and $\bar{\mu}$ is the effective viscosity of the porous medium and $\tilde{\mu}_{d}$ is the Darcian velocity which is related to the fluid velocity $\tilde{u}$ by $u_{d}=\tilde{u} \emptyset,(0<\emptyset<1)$ being the porosity of the medium. This assumes the same velocity in each pore, and the fluid in the pores is averaged over the volume. In general, the effective viscosity $\bar{\mu}$ and the fluid viscosity $\mu$ are different. However, at the macrolevel we may take them equal, though this assumption does not hold at the microlevel. Following Naduvinamani et al. [12] and Breugem [29], (2.3) can now be written as

$$
r=-\frac{\mu \emptyset \tilde{u}_{d}}{k}
$$

Continuity equation (2.1) implies the existence of a stream function $\psi(r, z)$ such that

$$
u_{r}(r, z)=\frac{1}{r} \frac{\partial \psi}{\partial r}, \quad u_{z}(r, z)=-\frac{1}{r} \frac{\partial \psi}{\partial r}
$$

Substitution of (2.5) together with (2.4) in (2.2) and elimination of pressure from the resulting equations using $\partial^{2} p / \partial x \partial y=\partial^{2} p / \partial y \partial x$ yield

$$
-\rho\left[\frac{\partial\left(\psi, E^{2} \psi / r\right)}{\partial(r, z)}\right]=\frac{\mu}{r} E^{4} \psi-\frac{\mu}{k} \frac{\mu E^{2} \psi}{r}
$$

where $E^{2}=\partial^{2} / \partial r^{2}-(1 / r)(\partial / \partial r)+\left(\partial^{2} / \partial z^{2}\right)$.

\section{Basic Idea of Differential Transform Method (DTM)}

If $F(z)$ is a given function, its differential transform is defined as

$$
\bar{F}(r)=\left.\frac{1}{r !} \frac{d^{r} F(z)}{d z^{r}}\right|_{z=0}
$$

The inverse transform of $\bar{F}(r)$ is defined by

$$
F(z)=\sum_{r=0}^{\infty} z^{r} \bar{F}(r)
$$


Table 1

\begin{tabular}{ll}
\hline Function & Transformed function \\
\hline$K(z)=\alpha K_{1}(z) \pm \beta K_{2}(z)$ & $K(r)=\alpha \bar{K}_{1}(r) \pm \beta \bar{K}_{2}(r)$ \\
$K(z)=F^{(m)}(z)$ & $K(r)=((m+r) ! / r !) F(m+r)$ \\
$K(z)=K_{1}(z) K_{2}(z)$ & $\bar{K}(r)=\sum_{n=0}^{r} \bar{K}_{1}(n) \bar{K}_{2}(r-n)$ \\
$K(z)=z^{m}$ & $\bar{K}(r)=\delta(r-m), \delta(p)=\left\{\begin{array}{l}1, p=0 \\
0, p \neq 0\end{array}\right.$ \\
$K(z)=K_{1}(z) K_{2}(z) \cdots K_{m}(z)$ & $G(r)=\sum_{l_{m-1=0}}^{r} \sum_{l_{m-2-2}}^{l_{m-1}} \cdots \sum_{l_{2=0}}^{l_{3}} \sum_{l_{1=0}}^{l_{2}}\left\{\bar{K}_{1}\left(l_{1}\right) \bar{K}_{2}\left(l_{2}-l_{1}\right) \cdots \bar{K}_{m}\left(r-l_{m-1}\right)\right.$ \\
\hline
\end{tabular}

In actual application, the function $F(z)$ is expressed by a finite series

$$
F(z)=\sum_{r=0}^{N} z^{r} \bar{F}(r)
$$

Equation (3.2) implies that

$$
F(z)=\sum_{r=N+1}^{\infty} z^{r} \bar{F}(r)
$$

is negligibly small.

The fundamental operations of the DTM are given in Table 1.

\section{Analysis of the Method}

Consider a fourth-order boundary value problem

$$
F^{(4)}(z)=G(z, F), \quad 0<z<H,
$$

with the following boundary conditions:

$$
F(0)=\alpha_{0}, \quad F(H)=\alpha_{1}, \quad F^{(1)}(H)=\alpha_{2}, \quad F^{(2)}(0)=\alpha_{3},
$$

where $\alpha_{i}, i=0,1,2,3$ are given values.

The differential transform of (4.1) is

$$
\bar{F}(r+4)=\frac{\bar{G}(r)}{\prod_{i=1}^{4}(r+i)}
$$

where $\bar{G}(r)$ is the differential transform of $G(z, F)$.

The transformed boundary conditions (4.2) are given by

$$
\bar{F}(0)=\alpha_{0}, \quad \sum_{r=0}^{N} H^{r} \bar{F}(r)=\alpha_{1}, \quad \sum_{r=0}^{N} r H^{r} \bar{F}(r)=\alpha_{2}, \quad \bar{F}(2)=\frac{\alpha_{3}}{2} .
$$


Table 2

\begin{tabular}{lcccc}
\hline$z$ & DTM & NDSolve & DTM residual & NDSolve residual \\
\hline 0 & 0 & 0 & 0 & $-5.106 \times 10^{-2}$ \\
0.1 & -0.018294487 & -0.01829449 & $-8.673 \times 10^{-18}$ & $-5.649 \times 10^{-3}$ \\
0.2 & -0.036260143 & -0.0362660146 & $-1.146 \times 10^{-14}$ & $1.243 \times 10^{-3}$ \\
0.3 & -0.053563634 & -0.053563633 & $-2.229 \times 10^{-12}$ & $4.938 \times 10^{-5}$ \\
0.4 & -0.069862544 & -0.069862646 & $-9.383 \times 10^{-11}$ & $-2.755 \times 10^{-4}$ \\
0.5 & -0.084800661 & -0.084800660 & $-1.706 \times 10^{-9}$ & $1.459 \times 10^{-4}$ \\
0.6 & -0.098003041 & -0.09800304 & $-1.826 \times 10^{-8}$ & $-1.237 \times 10^{-5}$ \\
0.7 & -0.109070812 & -0.109070809 & $-1.35 \times 10^{-7}$ & $-1.475 \times 10^{-4}$ \\
0.8 & -0.117575636 & -0.117575632 & $-7.683 \times 10^{-7}$ & $6.424 \times 10^{-4}$ \\
0.9 & -0.123053816 & -0.123053811 & $-3.550 \times 10^{-6}$ & $-2.699 \times 10^{-3}$ \\
1.0 & -0.125000000 & -0.124999996 & $1.395 \times 10^{-5}$ & $-3.186 \times 10^{-2}$ \\
\hline
\end{tabular}

Using (4.3) and (4.4), values of $\bar{F}(i), i=4,5, \ldots$ are obtained which give the following series solution up to $O\left(z^{N+1}\right)$,

$$
F(z)=\sum_{r=0}^{N} z^{r} \bar{F}(r)+O\left(z^{N+1}\right)
$$

\section{Our Problem}

Here, we consider an incompressible Newtonian fluid, squeezed between two large planar, parallel plates which are separated by a small distance $2 \mathrm{H}$ and moving towards each other with velocity $V$. For small values of the velocity $V$, the gap distance $2 H$ between the plates changes slowly with time $t$, so that it can be taken as constant, the flow is steady [30,31]:

$$
F^{(i v)}(z)-m^{2} F^{\prime \prime}(z)+2 R F(z) F^{\prime \prime \prime}(Z)=0,
$$

with the following boundary conditions:

$$
F(0)=0, \quad F^{(2)}(0)=0, \quad F(H)=-\frac{V}{2}, \quad F^{(1)}(H)=0,
$$

where $\sqrt{k / \mu}, R=\rho / \mu$.

The transformed boundary conditions (5.2) are

$$
\bar{F}(0)=0, \quad \bar{F}(1)=a, \quad \bar{F}(2)=0, \quad \bar{F}(3)=b,
$$

where $a$ and $b$ will be determined later.

The differential transform of (5.1) is

$$
\bar{F}(r+4)=\frac{r !}{(r+4) !}\left\{(r+1)(r+2) \bar{F}(r+2)-2 R \sum_{k=0}^{r}(k+1)(k+2)(k+3) \bar{F}(k+3) \bar{F}(r-k)\right\} .
$$


Table 3

\begin{tabular}{lcccccc}
\hline$z$ & $\begin{array}{c}k=1, \rho=0.8, \\
\mu=1\end{array}$ & $\begin{array}{c}k=0.5, \rho=0.9, \\
\mu=5\end{array}$ & $\begin{array}{c}k=0.1, \rho=1, \\
\mu=10\end{array}$ & $\begin{array}{c}k=0.1, \rho=0.8, \\
\mu=1\end{array}$ & $\begin{array}{c}k=1, \rho=1, \\
\mu=10\end{array}$ & $\begin{array}{c}k=0.5, \rho=1, \\
\mu=1000\end{array}$ \\
\hline 0 & 0 & 0 & 0 & 0 & 0 & 0 \\
0.1 & $-2.220 \times 10^{-16}$ & $6.939 \times 10^{-18}$ & 0 & $-5.551 \times 10^{-17}$ & 0 & $-1.084 \times 10^{-19}$ \\
0.2 & $-1.716 \times 10^{-12}$ & $2.470 \times 10^{-15}$ & $4.025 \times 10^{-16}$ & $-8.640 \times 10^{-13}$ & $4.163 \times 10^{-16}$ & $-3.253 \times 10^{-19}$ \\
0.3 & $-3.361 \times 10^{-10}$ & $4.476 \times 10^{-12}$ & $7.697 \times 10^{-14}$ & $-1.675 \times 10^{-10}$ & $7.978 \times 10^{-14}$ & $-3.253 \times 10^{-19}$ \\
0.4 & $-1.427 \times 10^{-8}$ & $2.016 \times 10^{-11}$ & $3.240 \times 10^{-12}$ & $-7.014 \times 10^{-9}$ & $3.367 \times 10^{-12}$ & $-4.337 \times 10^{-19}$ \\
0.5 & $-2.626 \times 10^{-7}$ & $3.699 \times 10^{-10}$ & $5.892 \times 10^{-11}$ & $-1.267 \times 10^{-7}$ & $6.143 \times 10^{-11}$ & $8.674 \times 10^{-19}$ \\
0.6 & $-2.849 \times 10^{-6}$ & $3.400 \times 10^{-9}$ & $6.303 \times 10^{-10}$ & $-1.343 \times 10^{-6}$ & $6.599 \times 10^{-10}$ & $1.01 \times 10^{-17}$ \\
0.7 & $-2.149 \times 10^{-5}$ & $3.005 \times 10^{-8}$ & $4.675 \times 10^{-9}$ & $-9.857 \times 10^{-6}$ & $4.198 \times 10^{-9}$ & $7.720 \times 10^{-17}$ \\
0.8 & $-1.243 \times 10^{-4}$ & $1.729 \times 10^{-7}$ & $2.652 \times 10^{-8}$ & $-5.520 \times 10^{-5}$ & $2.805 \times 10^{-8}$ & $4.402 \times 10^{-16}$ \\
0.9 & $-5.871 \times 10^{-4}$ & $8.125 \times 10^{-7}$ & $1.226 \times 10^{-7}$ & $-2.513 \times 10^{-4}$ & $1.304 \times 10^{-7}$ & $2.026 \times 10^{-15}$ \\
1.0 & $-2.367 \times 10^{-3}$ & $3.253 \times 10^{-6}$ & $4.821 \times 10^{-7}$ & $-9.705 \times 10^{-4}$ & $5.168 \times 10^{-7}$ & $7.914 \times 10^{-15}$ \\
\hline
\end{tabular}

Using (5.3) and (5.4), we obtain the following values of $\bar{F}(i), i=1,2,3, \ldots 15$ :

$$
\begin{gathered}
\bar{F}(2 k)=0, \quad \text { for } k=0(1), \\
\bar{F}(5)=\frac{1}{120}\left(6 b m^{2}-12 a b \operatorname{Re}\right), \\
\bar{F}(7)=\frac{1}{840}\left\{20 m^{2} \bar{F}(5)-2 \operatorname{Re}\left(6 b^{2}+60 a \bar{F}(5)\right)\right\}, \\
\bar{F}(9)=\frac{1}{3024}\left\{42 m^{2} \bar{F}(7)-2 \operatorname{Re}(66 b \bar{F}(5)+210 a \bar{F}(7))\right\}, \\
\bar{F}(11)=\frac{1}{7920}\left\{72 m^{2} \bar{F}(9)-2 \operatorname{Re}\left(60 \bar{F}^{2}(5)+216 b \bar{F}(7)+504 a \bar{F}(9)\right)\right\}, \\
\bar{F}(13)=\frac{1}{17160}\left\{110 m^{2} \bar{F}(11)-2 \operatorname{Re}(270 \bar{F}(5) \bar{F}(7)+510 b \bar{F}(9)+990 a \bar{F}(11))\right\}, \\
\bar{F}(15)=\frac{1}{37260}\left\{156 m^{2} \bar{F}(13)-2 \operatorname{Re}\left(210 \bar{F}^{2}(7)+564 \bar{F}(5) \bar{F}(9)+996 b \bar{F}(11)+171 a \bar{F}(13)\right)\right\} .
\end{gathered}
$$

We fix $H=1$ and $V=0.25$. The permeability $k=1$ and the fluid parameters $\mu=1, \rho=$ 1 are taken without units as they appear in the value of $m$ and $R$ which have been nondimensionalized. For these values, the unknowns $a$ and $b$ are determined by the following system:

$$
\mu \sum_{r=0}^{15} r(H)^{r-1} \bar{F}(r)=0, \quad \sum_{r=0}^{15} r(H)^{r} \bar{F}(r)=\frac{-V}{2} .
$$

We find that $a=-0.183491427, b=0.054618193$. 
Table 4

\begin{tabular}{lcc}
\hline$z$ & $H=1, V=0.5$ & $H=0.5, V=1$ \\
\hline 0 & 0 & 0 \\
0.1 & $-2.082 \times 10^{-17}$ & $-1.859 \times 10^{-12}$ \\
0.2 & $-2.047 \times 10^{-14}$ & $-1.492 \times 10^{-8}$ \\
0.3 & $-3.991 \times 10^{-11}$ & $-2.799 \times 10^{-6}$ \\
0.4 & $-1.684 \times 10^{-10}$ & $-1.115 \times 10^{-4}$ \\
0.5 & $-3.072 \times 10^{-8}$ & $-1.874 \times 10^{-3}$ \\
0.6 & $-3.299 \times 10^{-7}$ & $X$ \\
0.7 & $-2.458 \times 10^{-6}$ & $X$ \\
0.8 & $-1.401 \times 10^{-6}$ & $X$ \\
0.9 & $-6.515 \times 10^{-5}$ & $X$ \\
1.0 & $-2.578 \times 10^{-4}$ & $X$ \\
\hline
\end{tabular}

Now, using the inverse differential transform, the following approximate solution of $O\left(z^{16}\right)$ is obtained:

$$
\begin{aligned}
\widetilde{F}(z)= & \sum_{k=0}^{15} z^{k} \bar{F}(r) \\
= & -0.183491427 z+0.054618193 z^{3}+0.003733107 z^{5}+0.000144123 z^{7} \\
& -3.226 \times 10^{-6} z^{9}-7.452 \times 10^{-7} z^{11}-2.701 \times 10^{-8} z^{13}+1.975 \times 10^{-9} z^{15} .
\end{aligned}
$$

Residual of the solution is

$$
\hbar=\frac{d^{4} \tilde{F}(z)}{d z^{4}}-\frac{d^{2} \widetilde{F}(z)}{d z^{2}}+2 \widetilde{F}(z) \frac{d^{3} \widetilde{F}(z)}{d z^{3}}
$$

In Table 2, numerical results of the DTM solution are compared with the results of Mathematica NDSolve solution. Residuals of both the solutions are given for comparison. List Interpolation is used for the construction of approximating polynomial of the numerical solution by NDSolve.

In Table 3, residuals of the DTM solutions have been evaluated for various values of $k$, $\mu, \rho$, keeping $H=1$ and $V=1$ fixed.

In Table 4 , we fix $k=1, \mu=1, \rho=1$ and vary $H, V$. The reliability of the solution can be seen by looking into the residual at different mesh points.

\section{Conclusion}

In this paper, we studied squeezing flow in a porous medium between two parallel plates. Flow pattern for various parameters of Newtonian fluids are derived. When the parameters $H, m$, and $R$ are fixed as 1 , and velocity of the plates is varied, different velocity profiles are obtained. It is clearly visible that when the velocity of the plates is increased the fluid velocity is increased (Figure 1). For $k \leq 1$, the variation in flow pattern is negligible but 


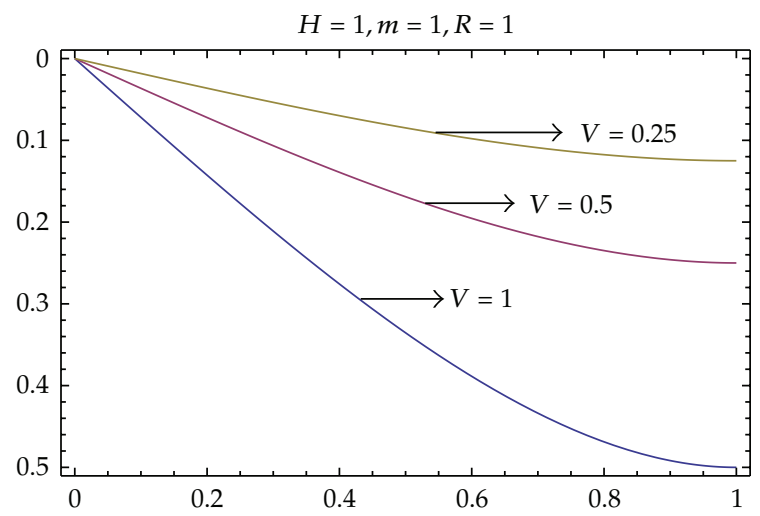

Figure 1: Velocity profiles of the squeezing flow in a porous medium when the plates velocity $V$ is varied.

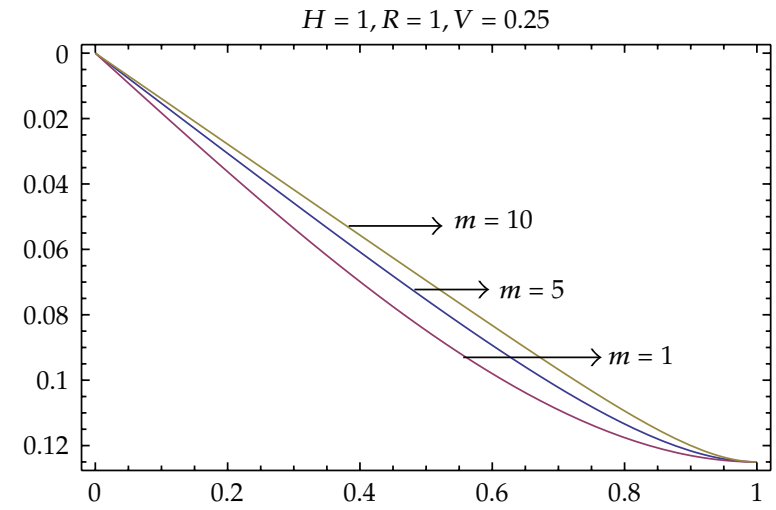

Figure 2: Velocity profiles of fluid flow when permeability $\kappa$, is increased for a fixed $\mu$.

for higher values of $k$ it does matter as seen in Table 2, and Figure 2. It is also noticed that at lower velocity of the plates the flow rate is also lower. The results obtained by the application of DTM are reliable with high accuracy. Applicability of the method is simple and needs no restrictions of large and small parameters. It avoids the difficulties and massive computational work encountered in other numerical techniques such linearization, discretization and perturbation. Moreover this method has superiority over the Adomian decomposition method as it does not require calculation of Adomian polynomials in case of nonlinearity. Furthermore, the solution obtained by this method converges rapidly to analytical solution in case of integrable system.

\section{Acknowledgment}

The authors would like to thank the anonymous reviewers for their helpful and encouraging comments on earlier draft of this paper.

\section{References}

[1] W. Tan and T. Masuoka, "Stokes' first problem for a second grade fluid in a porous half-space with heated boundary," International Journal of Non-Linear Mechanics, vol. 40, no. 4, pp. 515-522, 2005. 
[2] J. Bear, Dynamics of Fluids in Porous Media, Elsevier, New York, NY, USA, 1972.

[3] H. C. Brinkman, "A calculation of the viscous force exerted by a flowing fluid on a dense swarm of particles," Applied Scientific Research, vol. 1, no. 1, pp. 27-34, 1947.

[4] M. H. Hamdan, "An alternative approach to exact solutions of a special class of Navier-Stokes flows," Applied Mathematics and Computation, vol. 93, no. 1, pp. 83-90, 1998.

[5] M. H. Hamdan and F. M. Allan, "A note on the generalized Beltrami flow through porous media," International Journal of Pure and Applied Mathematics, vol. 27, no. 4, pp. 491-500, 2006.

[6] K. Vafai and C. L. Tien, "Boundary and inertia effects on flow and heat transfer in porous media," International Journal of Heat and Mass Transfer, vol. 24, no. 2, pp. 195-203, 1981.

[7] S. Islam, M. R. Mohyuddin, and C. Y. Zhou, "Few exact solutions of non-Newtonian fluid in porous medium with hall effect," Journal of Porous Media, vol. 11, no. 7, pp. 669-680, 2008.

[8] S. Islam and C. Y. Zhou, "Certain inverse solutions of a second-grade magnetohydrodynamic aligned fluid flow in a porous medium," Journal of Porous Media, vol. 10, no. 4, pp. 401-408, 2007.

[9] K. Vafai, Hand Book of Porous Media, CRC Press, Taylor \& Francis Group, Boca Raton, Fla, USA, 2nd edition, 2005.

[10] A. S. Burbidge and C. Servais, "Squeeze flows of apparently lubricated thin films," Journal of NonNewtonian Fluid Mechanics, vol. 124, no. 1-3, pp. 115-127, 2004.

[11] M. J. Stefan, "Versuch Uber die scheinbare adhesion," Akademie der Wissenschaften in Wien. Mathematisch-Naturwissenschaftliche, vol. 69, p. 713, 1874.

[12] N. B. Naduvinamani, P. S. Hiremath, and G. Gurubasavaraj, "Static and dynamic behaviour of squeeze-film lubrication of narrow porous journal bearings with coupled stress fluid," Proceedings of the Institution of Mechanical Engineers, Part J: Journal of Engineering Tribology, vol. 215, no. 1, pp. 45-62, 2001.

[13] N. B. Naduvinamani, P. S. Hiremath, and G. Gurubasavaraj, "Squeeze film lubrication of a short porous journal bearing with couple stress fluids," Tribology International, vol. 34, no. 11, pp. 739-747, 2001.

[14] Q. K. Ghori, M. Ahmed, and A. M. Siddiqui, "Application of homotopy perturbation method to squeezing flow of a newtonian fluid," International Journal of Nonlinear Sciences and Numerical Simulation, vol. 8, no. 2, pp. 179-184, 2007.

[15] X. J. Ran, Q. Y. Zhu, and Y. Li, "An explicit series solution of the squeezing flow between two infinite plates by means of the homotopy analysis method," Communications in Nonlinear Science and Numerical Simulation, vol. 14, no. 1, pp. 119-132, 2009.

[16] R. J. Grimm, "Squeezing flows of Newtonian liquid films an analysis including fluid inertia," Applied Scientific Research, vol. 32, no. 2, pp. 149-166, 1976.

[17] W. F. Hughes and R. A. Elco, "Magnetohydrodynamic lubrication flow between parallel rotating disks," Journal of Fluid Mechanics, vol. 13, pp. 21-32, 1962.

[18] S. Kamiyama, "Inertia Effects in MHD hydrostatic thrust bearing," ASME Transactions Journal of Lubrication Technology, vol. 91, pp. 589-596, 1969.

[19] E. A. Hamza, “The Magnetohydrodynamic squeeze film,” Journal of Tribology, vol. 110, no. 2, pp. 375$377,1988$.

[20] S. Bhattacharyya and A. Pal, "Unsteady MHD squeezing flow between two parallel rotating discs," Mechanics Research Communications, vol. 24, no. 6, pp. 615-623, 1997.

[21] J. K. Zhou, Deferential Transformation and Its Application for Electrical Circuits, Huazhong University Press, Wuhan, China, 1986.

[22] F. Ayaz, "Applications of differential transform method to differential-algebraic equations," Applied Mathematics and Computation, vol. 152, no. 3, pp. 649-657, 2004.

[23] H. Liu and Y. Song, "Differential transform method applied to high index differential-algebraic equations," Applied Mathematics and Computation, vol. 184, no. 2, pp. 748-753, 2007.

[24] F. Ayaz, "On the two-dimensional differential transform method," Applied Mathematics and Computation, vol. 143, pp. 361-374, 2003.

[25] I. H. A. Hassan, "Comparison deferential transformation technique with Adomian decomposition method for linear and nonlinear initial value problems," Chaos, Solution and Fractals, vol. 36, no. 1, pp. 53-65, 2007.

[26] S. U. Islam, S. Haq, and J. Ali, "Numerical solution of special 12th-order boundary value problems using differential transform method," Communications in Nonlinear Science and Numerical Simulation, vol. 14, no. 4, pp. 1132-1138, 2009.

[27] M. T. Kamel and H. M. Hamdan, "Riabounchisky flow through porous media," International Journal of Pure and Applied Mathematics, vol. 27, no. 1, pp. 113-126, 2006. 
[28] N. Merabet, H. Siyyam, and M. H. Hamdan, "Analytical approach to the Darcy-Lapwood-Brinkman equation," Applied Mathematics and Computation, vol. 196, no. 2, pp. 679-685, 2008.

[29] W. -P. Breugem, "The effective viscosity of a channel-type porous medium," Physics of Fluids, vol. 19, no. 10, 2007.

[30] T. C. Papanastasiou, G. C. Georgio, and A. N. Alexandrou, Viscous Fluid Flow, CRC Press, New York, NY, USA, 1994.

[31] M. Idrees, S. Islam, S. Haq, and S. Islam, "Application of the optimal homotopy asymptotic method to squeezing flow," Computers and Mathematics with Applications, vol. 59, no. 12, pp. 3858-3866, 2010. 


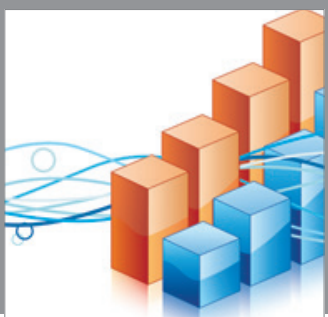

Advances in

Operations Research

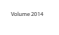

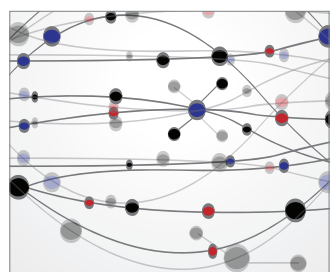

\section{The Scientific} World Journal
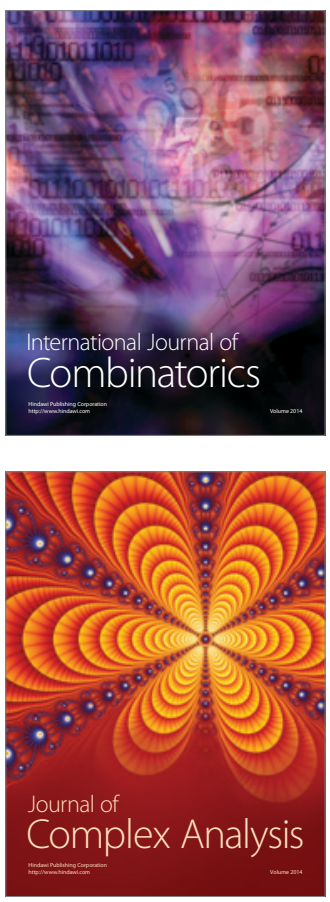

International Journal of

Mathematics and

Mathematical

Sciences
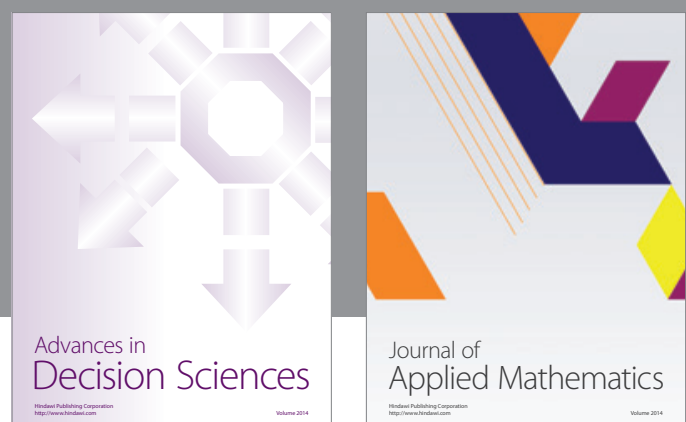

Journal of

Applied Mathematics
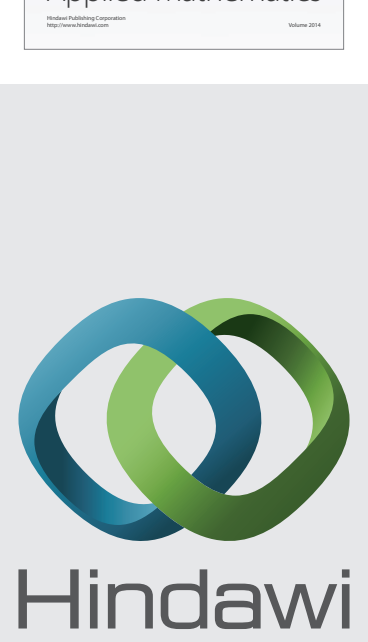

Submit your manuscripts at http://www.hindawi.com
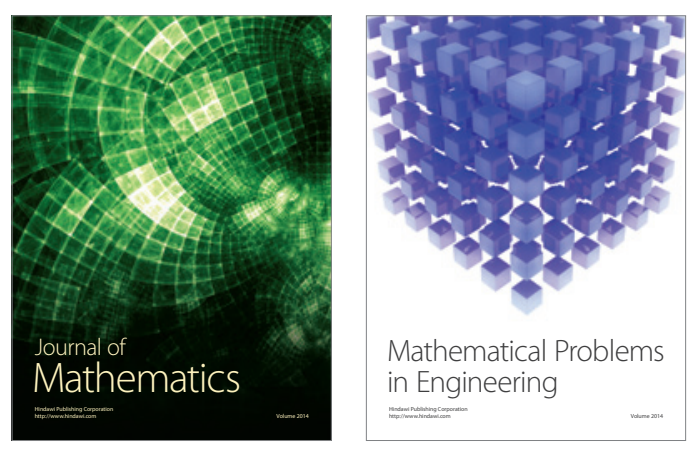

Mathematical Problems in Engineering
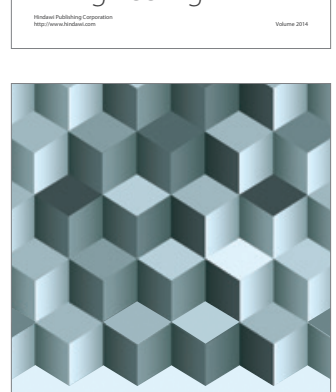

Journal of

Function Spaces
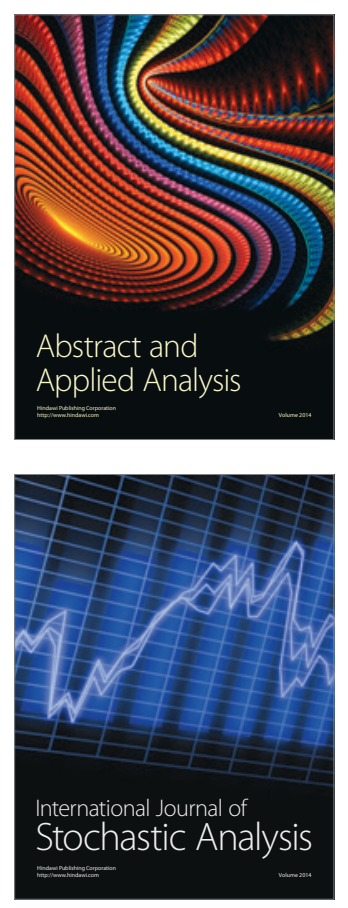

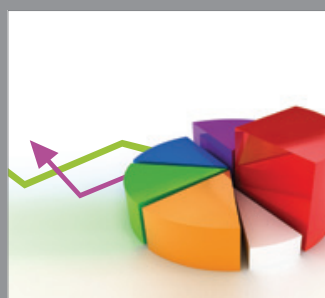

ournal of

Probability and Statistics

Promensencen
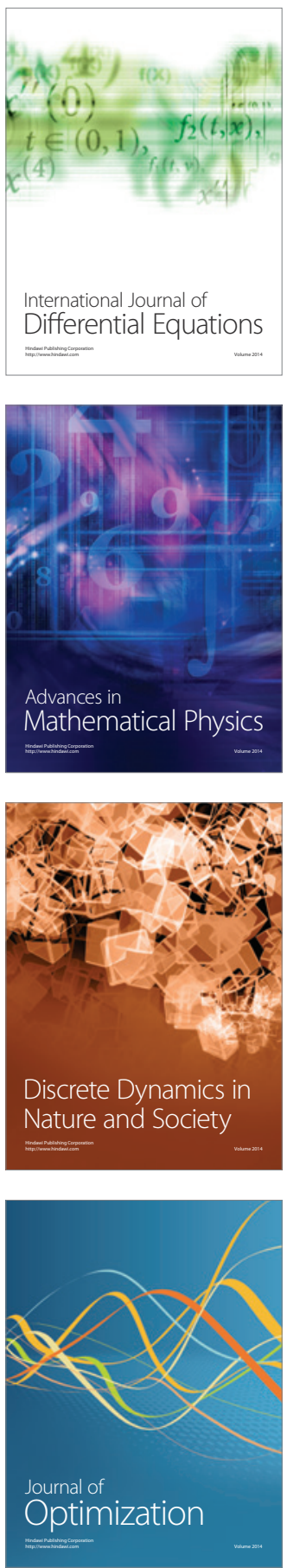